\title{
PERFECT COLOURINGS OF CYCLOTOMIC INTEGERS
}

\author{
E.P. BUGARIN, M.L.A.N. DE LAS PEÑAS, AND D. FRETTLÖH
}

\begin{abstract}
Perfect colourings of the rings of cyclotomic integers with class number one are studied. It is shown that all colourings induced by ideals $(q)$ are chirally perfect, and vice versa. A necessary and sufficient condition for a colouring to be perfect is obtained, depending on the factorisation of $q$. This result yields the colour symmetry group $H$ in general. Furthermore, the colour preserving group $K$ is determined in all but finitely many cases. An application to colourings of quasicrystals is given.
\end{abstract}

\section{INTRODUCTION}

The study of colour symmetries of periodic patterns or point lattices in two or three dimensions is a classical topic, see 8 or 13 . A colour symmetry is a symmetry of a coloured pattern up to permutation of colours; and the study of colour symmetry groups is dedicated to the relation of the colour symmetries of a coloured pattern to the symmetries of the uncoloured pattern. During the last century, the classification of colour symmetry groups of periodic patterns has been carried out to a great extent. The discovery of quasiperiodic patterns [15] like the Penrose tiling raised the question about colour symmetries of these patterns. Quasiperiodic patterns are not periodic, that is, the only translation fixing the pattern is the trivial translation by 0 . Nevertheless, quasiperiodic patterns show a high degree of short and long range order. One early approach to generalise the concept of colour symmetry to quasiperiodic patterns was given in [9]. It used the notion of indistinguishability of coloured patterns and the fact that, for quasiperiodic patterns, it can be described in Fourier space rather than in real space [7. A more algebraic approach was used in [12, making use of quadratic number fields. In this work, the problem of colour symmetries of both periodic and non-periodic patterns, including the quasiperiodic cases, is addressed by studying the sets of cyclotomic integers following the setting introduced in [1, [2], 3. Cyclotomic integers turned out to be very useful in describing symmetries of quasiperiodic patterns.

This article can be seen as a complement to [2, which concentrates on the combinatorial aspects of perfect or chirally perfect colourings of $\mathcal{M}_{n}$, where $\mathcal{M}_{n}=\mathbb{Z}\left[e^{2 \pi i / n}\right]$ denotes a $\mathbb{Z}$-module of cyclotomic integers. (To be precise, they study so called 'Bravais colourings', but we will show in the sequel that Bravais colourings and chirally perfect colourings are the same.) In particular, the results in 2] yield the numbers $\ell$ for which a (chirally) perfect colouring of $\mathcal{M}_{n}$ with $\ell$ colours exists, given that $\mathcal{M}_{n}$ has class number one. In contrast, this paper studies the algebraic properties of the colour symmetry groups of perfect colourings of $\mathcal{M}_{n}$, again for the case that $\mathcal{M}_{n}$ has class number one. In this sense this paper follows the spirit of [5], where the group structure arising from of colourings (not necessariliy perfect ones) of periodic patterns are studied. Finally we should mention that the methods of this paper had been applied to a more detailed study of perfect colourings of cyclotomic integers with 5 -fold, 8 -fold and 12 -fold symmetry.

\section{Preliminaries}

Let $\mathcal{M}_{n}$ denote the $n$-th ring of cyclotomic integers. That is, $\mathcal{M}_{n}=\mathbb{Z}\left[\xi_{n}\right]$ is the ring of polynomials in $\xi_{n}$, where $\xi_{n}=e^{2 \pi i / n}$ always is a primitive complex root of unity. If it is clear from the context, we may write just $\xi$ instead of $\xi_{n}$. Since $\mathcal{M}_{2 n}=\mathcal{M}_{n}$ for $n$ odd, we omit the case $n \equiv 2 \bmod 4$, for the sake of uniqueness. As mentioned above, our approach requires that $\mathcal{M}_{n}$ has class number 
one. Then we can use the fact that $\mathcal{M}_{n}$ is a principal ideal domain, and therefore also a unique factorisation domain. This is only true for the following values of $n$.

(1)

$$
n=3,4,5,7,8,9,11,12,13,15,16,17,19,20,21,24,25,27,28,32,33,35,36,40,44,45,48,60,84 .
$$

Let us emphasise that $\mathcal{M}_{n}$ always denotes the ring of cyclotomic integers for the values in Equation (1) only.

Notation: Throughout the text, $D_{n}$ (resp. $C_{n}$ ) denotes the dihedral (resp. cyclic) group of order $2 n$ (resp. $n$ ). The symmetric group of order $n$ ! is denoted by $\mathcal{S}_{n}$. Let $\xi_{n}=e^{2 \pi i / n}$, a primitive $n$-th root of unity. The set of cyclotomic integers $\mathbb{Z}\left[\xi_{n}\right]$ is denoted by $\mathcal{M}_{n}$. The point group of $\mathcal{M}_{n}$ (the set of linear isometries fixing $\mathcal{M}_{n}$ ) is the dihedral group $D_{N}$, where $N=n$ if $n$ is even, and $N=2 n$ if $n$ is odd. The entire symmetry group $G\left(\mathcal{M}_{n}\right)$ of $\mathcal{M}_{n}$ is symmorphic, that is, it equals the semidirect product of its translation subgroup with its point group: $G\left(\mathcal{M}_{n}\right)=\mathcal{M}_{n} \rtimes D_{N}$, where $N=n$ if $n$ is even, and $N=2 n$ if $n$ is odd. If $H$ is a subgroup of some group $G$, the index of $H$ in $G$ is denoted by $[G: H]$. Throughout the text we will identify the Euclidean plane with the complex plane. The complex norm of $z \in \mathbb{C}$ is always denoted by $|z|$, while the algebraic norm of $z \in \mathcal{M}_{n}$ is denoted by $N_{n}(z)$.

The symmetry group of some set $X \subset \mathbb{R}^{2}$ is always denoted by $G$ in the sequel. The following definitions are mainly taken from [8]. A colouring of $X$ is a surjective map $c: X \rightarrow\{1, \ldots, \ell\}$. Whenever we want to emphasise that a colouring uses $\ell$ colours, we will also call it an $\ell$-colouring. The objects of interest are colourings where an element of $G$ acts as a global permutation of the colours. Thus, for given $X \subset \mathbb{R}^{2}$ and a colouring $c$ of $X$, we consider the following group.

$$
H=\left\{h \in G \mid \exists \pi \in \mathcal{S}_{\ell} \forall x \in X: c(h(x))=\pi(c(x))\right\} .
$$

The elements of $H$ are called colour symmetries of $X . H$ is the colour symmetry group of the coloured pattern $(X, c)$.

Definition 2.1. A colouring $c$ of a point set $X$ is called perfect, if $H=G$. It is called chirally perfect, if $H=G^{\prime}$, where $G^{\prime}$ is the index 2 subgroup of $G$ containing the orientation preserving isometries in $G$.

See Figure 1 for some examples. By the requirement $\pi c=c h$, each $h$ determines a unique permutation $\pi=\pi_{h}$. This also defines a map

$$
P: H \rightarrow \mathcal{S}_{\ell}, \quad P(h):=\pi_{h} .
$$

Let $g, h \in H$. Because of $c(h g(x))=\operatorname{ch}(g(x))=\pi_{h} c(g(x))=\pi_{h}\left(\pi_{g}(c(x))\right)=\pi_{h} \pi_{g}(c(x))$, we obtain the following result.

Lemma 2.2. $P$ is a group homomorphism.

A further object of interest is the subgroup $K$ of $H$ which fixes the colours. For a given $X \subset \mathbb{R}^{2}$ and a colouring $c$ of $X$, we consider the colour preserving group $K$ (in 4] called colour fixing group):

$$
K:=\{k \in H \mid c(k(x))=c(x), x \in X\} .
$$

In other words, $K$ is the kernel of $P$. The aim of this paper is to deduce the nature of the groups $H$ and $K$ for (chirally) perfect colourings of $\mathcal{M}_{n}$.

\section{Coset COLOURINGS AND IDEAL COLOURINGS OF PLANAR MOdUleS}

A colouring of a point set $X$ with a group structure (like a lattice or a $\mathbb{Z}$-module) can be constructed by choosing a subgroup of $X$ and assigning to each coset a different colour ([16, see also [6]). Thus we will generate colourings of $\mathcal{M}_{n}$ by suitable subgroups of $\mathcal{M}_{n}$. Since $\mathcal{M}_{n}$ is in fact a principal ideal domain, we will choose principal ideals $(q)$ as these subgroups. Each element $q \in \mathcal{M}_{n}$ thus generates a colouring in the following way. 

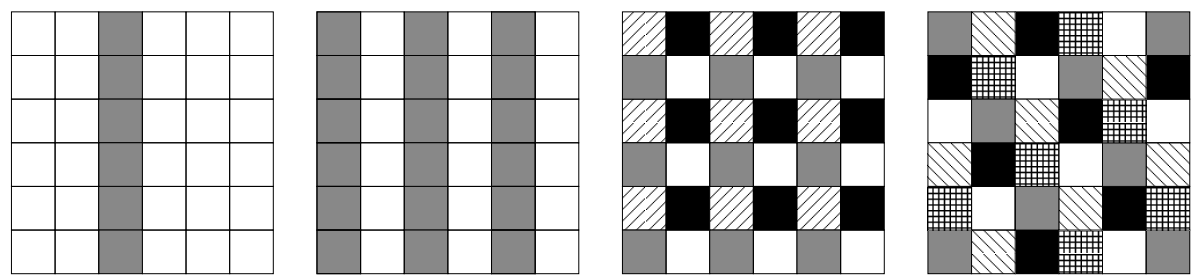

FiguRE 1. Four examples of colourings of $\mathbb{Z}^{2}$. For clarity, each element of $\mathbb{Z}^{2}$ is replaced by a unit square. From left to right: An arbitrary 2-colouring with two colours, neither ideal nor perfect; a 2-colouring induced by a coset colouring, but neither ideal nor perfect; a perfect 4-colouring induced by the ideal (2); a chirally perfect 5 -colouring induced by the ideal $(2+i)$.

Definition 3.1. An ideal colouring of $\mathcal{M}_{n}$ with $\ell$ colours is defined as follows: For each $z \in(q)=$ $q \mathcal{M}_{n}$, let $c(z)=1$. Let the other cosets of $(q)$ be $(q)+t_{2}, \ldots,(q)+t_{\ell}$. For each $z \in(q)+t_{i}$, let $c(z)=i$.

If $(q)$ is given explicitly, we will also call such an ideal colouring a colouring induced by $(q)$. We will see that all chirally perfect colourings of $\mathcal{M}_{n}$, where $n$ is of class number one, arise from principal ideals $(q)=q \mathcal{M}_{n}$, where $q \in \mathcal{M}_{n}$. Consequently, there exists a chirally perfect colouring of $\mathcal{M}_{n}$ with $\ell$ colours, if and only if there is $q$ such that $N_{n}(q)=\left[\mathcal{M}_{n}:(q)\right]=\ell$. (Note that the index of $(q)$ in $\mathcal{M}_{n}$ is just the algebraic norm of $q$.)

In [2], the number of Bravais colourings of $\mathcal{M}_{n}$ was obtained for all $n$ as in (11). Let us shortly explain, why in this context Bravais colourings are chirally perfect colourings, and vice versa. A Bravais colouring of $\mathcal{M}_{n}$ is a colouring where each one-coloured subset is in the same Bravais class as $\mathcal{M}_{n}$. In plain words, this means that each one-coloured subset is similar to $\mathcal{M}_{n}$. More precisely: there is $q \in \mathbb{C}$ such that for each $i, c^{-1}(i)$ is a translate of $q \mathcal{M}_{n}$. For a general definition of Bravais class, see for instance [11].

Theorem 3.2. Let $\mathcal{M}_{n}=\mathbb{Z}\left[\xi_{n}\right]$ be a principal ideal domain. A colouring of $\mathcal{M}_{n}$ is a Bravais colouring, if and only if it is a chirally perfect colouring, if and only if it is an ideal colouring.

Proof. Let $c$ be an ideal colouring induced by $(q)$. Trivially, $(q)=q \mathcal{M}_{n}$ is similar to $\mathcal{M}_{n}$, and the cosets are translates of $q \mathcal{M}_{n}$. Thus $c$ is a Bravais colouring.

Let $c$ be a Bravais colouring of $\mathcal{M}_{n}$. Without loss of generality, let $0 \in c^{-1}(1)$. The set $c^{-1}(1)$ of points of colour 1 is similar to $\mathcal{M}_{n}$, that is, it equals $q \mathcal{M}_{n}$ for some $q \in \mathbb{C}$. Since $c^{-1}(1) \subset \mathcal{M}_{n}$, we have $q \mathcal{M}_{n} \subset \mathcal{M}_{n}$, which implies $q \in \mathcal{M}_{n}$. Thus $c^{-1}(1)=(q)$. All other preimages $c^{-1}(i)$ are translates of $(q)$, thus cosets of $(q)$ in $\mathcal{M}_{n}$. Therefore $c$ is an ideal colouring.

For the equivalence of chirally perfect colouring and ideal colouring, see Theorem 4.4 below.

\section{The Structure of $H$}

Recall that $P: H \rightarrow \mathcal{S}_{\ell}$ maps a colour symmetry to the permutation it induces on the colours, see (3).

Lemma 4.1. $H$ acts transitively on the coloured subsets of any perfect colouring of $\mathcal{M}_{n}$, and $H / K \cong P(H)$.

Proof. The proof of the first statement follows from the proof of Theorem 4.4 below, see the remark there. Since $K=\operatorname{ker}(P)$, the second claim is clear.

This yields the short exact sequence

$$
0 \longrightarrow K \longrightarrow H \longrightarrow H / K \longrightarrow 0 .
$$


Therefore, $H$ is always a group extension of $K$. In general, $H$ is neither a direct nor a semidirect product of $K$ and $H / K$, see Theorem 4.7 below.

We proceed by examining how the factorisation of $q$ in $\mathcal{M}_{n}$ affects the structure of the colour symmetry group $H$ of the colouring induced by $(q)$. The unique factorisation of $q$ over $\mathcal{M}_{n}$ reads

$$
q=\varepsilon \prod_{p_{i} \in \mathcal{P}} p_{i}^{\alpha_{i}} \prod_{p_{j} \in \mathcal{C}} \omega_{p_{j}}^{\beta_{j}}{\overline{\omega_{p_{j}}}}^{\gamma_{j}} \prod_{p_{k} \in \mathcal{R}} p_{k}^{\delta_{k}},
$$

where $\varepsilon$ is a unit in $\mathcal{M}_{n}$. Here, $\mathcal{P}$ (resp. $\mathcal{C}$, resp. $\mathcal{R}$ ) denotes the set of inert (resp. complex splitting, resp. ramified) primes over $\mathcal{M}_{n}$. The generator $q$ is called balanced if $\beta_{j}=\gamma_{j}$ for all $j$. In other words: $q$ is balanced if it is of the form

$$
q=\varepsilon x p,
$$

where $\varepsilon$ is a unit in $\mathcal{M}_{n}, x$ is a real number in $\mathcal{M}_{n}$ (i.e., $x \in \mathbb{Z}[\xi+\bar{\xi}]$ ), and $p$ is a product of ramified primes. By the definition of a ramified prime $p$ (see [17]), $\bar{p} \in(p)$ holds in $\mathcal{M}_{n}$. (Equivalently, $p / \bar{p}$ is a unit in $\mathcal{M}_{n}$.) The following lemma is well-known, it is stated here for the convenience of the reader.

Lemma 4.2. All units $\varepsilon$ in $\mathbb{Z}\left[\xi_{n}\right]$ are of the form $\varepsilon= \pm \lambda \xi_{n}^{k}$, where $\lambda \in \mathbb{Z}[\xi+\bar{\xi}]$.

Proof. (Essentially [17], Prop. 1.5:) Let $\varepsilon$ be a unit, and let $\alpha=\varepsilon / \bar{\varepsilon}$. Since $\varepsilon, \bar{\varepsilon}, 1 / \bar{\varepsilon} \in \mathcal{M}_{n}, \alpha$ is an algebraic integer. Since complex conjugation commutes with any element of the Galois group, for all algebraic conjugates $\alpha_{i}$ of $\alpha$ holds $\left|\alpha_{i}\right|=1$.

From Lemma 1.6 of [17] then follows: If for all algebraic conjugates $\alpha_{i}$ of $\alpha$ holds $\left|\alpha_{i}\right|=1$, and $\alpha$ is an algebraic integer, then $\alpha$ is some root of unity, say, $\xi_{r}^{q}$. Since $\alpha \in \mathcal{M}_{n}$, it is either an $n$-th root of unity, or a $2 n$-th root of unity, if $n$ is odd. In each case, $\alpha= \pm \xi_{n}^{j}$ for some $j$. Then $\varepsilon^{2}=\varepsilon \bar{\varepsilon} \alpha=|\varepsilon| \xi_{n}^{j}$, where $|\varepsilon|$ is a real number, thus $|\varepsilon| \in \mathbb{Z}\left[\xi_{n}+\bar{\xi}_{n}\right]$. It follows $\varepsilon=\sqrt{|\varepsilon|} \xi_{n}^{j / 2}= \pm \sqrt{|\varepsilon|} \xi_{2 n}^{j}= \pm \lambda \xi_{2 n}^{j}$, where $\lambda \in \mathbb{Z}\left[\xi_{n}+\bar{\xi}_{n}\right]$. However, since $\varepsilon \in \mathbb{Z}\left[\xi_{n}\right]$, it can't be a proper $2 n$-th complex root of unity. Thus $\pm \lambda \xi_{2 n}^{j}= \pm \lambda \xi_{n}^{k}$ for some $k$.

In particular, if $\varepsilon$ is a unit in $\mathbb{Z}\left[\xi_{n}\right]$ with $|\varepsilon|=1$, then it is (up to sign) an $n$-th complex root of unity: $\varepsilon= \pm \xi_{n}^{k}$.

Lemma 4.3. $\bar{q} \in(q)$ if and only if $q$ is balanced.

Proof. Let $q$ be as in (6). Consider $q / \bar{q}$. The inert primes in numerator and denominator cancel each other. The unit $\varepsilon$, as well as the factors of the ramified primes, contribute a unit $\varepsilon^{\prime} \in \mathcal{M}_{n}$. Thus

$$
r:=q / \bar{q}=\varepsilon^{\prime} \prod_{p_{j} \in \mathcal{C}} \omega_{p_{j}}^{\beta_{j}-\gamma_{j}}{\overline{\omega_{p_{j}}}}^{\gamma_{j}-\beta_{j}} .
$$

If $q$ is balanced, then $\beta_{j}=\gamma_{j}$, thus $\bar{q}=\left(\varepsilon^{\prime}\right)^{-1} q \in(q)$, since $\varepsilon^{\prime}$ is a unit. If $q$ is not balanced, then $\beta_{j}-\gamma_{j} \neq 0$ for some $j$. Then, by Lemma 4.2, the right hand side $r$ is not a unit, thus $r^{-1} \notin \mathcal{M}_{n}$, and consequently $\bar{q}=r^{-1} q \notin(q)$.

Theorem 4.4. Let $\mathcal{M}_{n}=\mathbb{Z}\left[\xi_{n}\right]$ be a principal ideal domain.

(1) Each chirally perfect colouring of $\mathcal{M}_{n}$ is an ideal colouring.

(2) Each ideal colouring of $\mathcal{M}_{n}$ is chirally perfect.

(3) The colouring $c$ induced by $(q)$ is perfect, if and only if $q$ is balanced.

Consequently, $H=\mathcal{M}_{n} \rtimes D_{N}$ in the latter case, and $H=\mathcal{M}_{n} \rtimes C_{N}$ otherwise. $(N=2 n$ if $n$ is odd, $N=n$ else.)

Proof. Let $(q)$ be the ideal inducing the colouring of $\mathcal{M}_{n}$. Let $\ell=\left[\mathcal{M}_{n}:(q)\right]$, and denote the cosets of $(q)$ by $(q)+t_{1}, \ldots,(q)+t_{\ell}$, where $t_{1}=0$ for convenience. (The notation $(q)+g$ rather than $g(q)$ or $(q) g$ is justified as follows: all rotations and reflections in $G$ fix $(q)$. Only maps with some translational part map $(q)$ to a coset different from $(q)$.) 
We proceed by studying whether an element $g \in G$ maps an entire coset $(q)+t_{i}$ to an entire coset $(q)+t_{j}$ or not. If yes, then $g$ induces a global permutation of the colours, and $g \in H$. Three cases have to be considered.

1. Let $g \in G$ be a translation. Then $g$ is of the form $g(x)=x+t$ for some $t$. Hence $g\left((q)+t_{i}\right)=$ $(q)+t_{i}+t$ trivially is a coset of $(q)$.

2. Let $g \in G$ be a rotation. Then $g((q))=(q)$, thus $g\left((q)+t_{j}\right)=(q)+g\left(t_{i}\right)$, which is again a coset of $(q)$.

So, the first two cases are not critical, whether $q$ is balanced or not. In particular, all orientation preserving isometries map entire cosets to entire cosets, which proves part (2) of Theorem 4.4.

3. Let $g \in G$ be the reflection $x \mapsto \bar{x}$. If $q$ is balanced, then, by Lemma 4.3. $g(q)=\bar{q} \in(q)$, hence $g((q))=(q)$. Thus $g\left((q)+t_{j}\right)=(q)+g\left(t_{j}\right)$, which is a coset of $(q)$. Any element of $G$ is a composition of the three symmetries above, hence the 'if'-part of Theorem 4.4 (3) follows.

If $q$ is not balanced, then $g(0)=0 \in(q)$, but $g(q) \notin(q)$ by Lemma 4.3 . Thus $g$ does not map entire cosets to entire cosets. Consequently, no reflection in $G$ maps entire cosets to entire cosets. The reflections in $G$ are the only elements which fail to do so. This (again) shows Theorem 4.4 (2), and the 'only-if' part of Theorem $4.4(3)$.

Regarding Theorem 4.4 (1): Let $c$ be a chirally perfect colouring. Let $0 \in c^{-1}(1)$. Then $c^{-1}(1)$ is invariant and closed under rotations in $C_{N}$, and under translations by $t \in c^{-1}(1)$. It follows that $c^{-1}(1)$ is invariant under multiplication by elements of $\mathcal{M}_{n}$, and under translations by elements of $c^{-1}(1)$. Thus $c^{-1}(1)$ is an ideal in $\mathcal{M}_{n}$.

This proves Lemma 4.1 as well: $H$ contains all translations $z \mapsto z+t, t \in \mathcal{M}_{n}$. Clearly, these translations act transitively on the cosets.

Corollary 4.5. If there exists only one ideal colouring of $\mathcal{M}_{n}$ with $\ell$ colours, the colouring is perfect.

Proof. If an ideal colouring induced by $(q)$ is not perfect, then $q$ is not balanced by Theorem 4.4 Thus, $\bar{q} \notin(q)$ by Lemma 4.3 , hence $(q) \neq(\bar{q})$. So $(q)$ and $(\bar{q})$ define two different colourings with $\ell$ colours.

Now we get immediately a result on colourings of $\mathbb{Z}^{2}$. This is Theorem 8.7.1 in [8], see also [14]. Note that the number of colours $\ell$ is the norm $N_{4}(q)$ of $q$, which is just $q \bar{q}$.

Corollary 4.6. Let $c$ be an $\ell$-colouring of the square lattice $\mathbb{Z}[i]$ generated by $(q), q \in \mathbb{Z}[i]$.

(1) If the factorisation of $\ell$ over $\mathbb{Z}$ contains no primes $p \equiv 1 \bmod 4$, then the colouring is perfect.

(2) If $q=m$, or $q=i m$, or $q=(1 \pm i)^{k} m$ for some $m, k \in \mathbb{Z} \backslash\{0\}$, then the colouring is perfect.

(3) Otherwise the colouring is not perfect but chirally perfect, and so $H=\mathcal{M}_{n} \rtimes C_{N}$.

Proof. The inert primes in $\mathcal{M}_{4}=\mathbb{Z}[i]$ are exactly the ones of the form $p \equiv 3 \bmod 4$; and the splitting primes are exactly those of the form $p \equiv 1 \bmod 4$. The only ramified prime in $\mathcal{M}_{4}$ is $2=(1+i)(1-i)$. So $(1)$ and $(2)$ of Corollary 4.6 cover exactly the cases where $q$ is balanced, and the claim follows from Theorem 4.4.

Corollary 4.6 tells us that all ideal colourings of the square lattice with $1,2,4,8,9,16$ or 18 colours are perfect, and all those with 5,10,13,17,20 colours are not. (These are all possible values for $\ell<25$, see [2]). The first ambiguity occurs at the value $\ell=25$ : the three possible generators are $q=5, q=3+4 i, q=3-4 i$. The first one induces a perfect colouring, whereas the other two induce non-perfect but chirally perfect colourings. 
Theorem 4.7. If $\ell=2$, then $H$ is a semidirect product: $H=K \rtimes H / K$. If $\ell>N$, then $H \neq K \rtimes H / K .(N=2 n$, if $n$ odd, $N=n$ else. $)$

Proof. Consider Equation (5). By the splitting lemma, $H=K \rtimes H / K$, if and only if there is a homomorphism $Q: H / K \rightarrow H$ such that $P Q=$ id on $H / K$. If $\ell=2$, then $H / K \cong C_{2}=\{$ id, $x\}$. Let $Q($ id $)=$ id and $Q(x)=\varphi$, where $\varphi$ is the reflection in the vertical line through $\frac{1}{2}$. Certainly, $\varphi \in G$ holds: $\varphi: a+b i \mapsto 1-a+b i$ is a composition of $z \mapsto \bar{z}, z \mapsto i z, z \mapsto z+1$. By Lemma 5.2 (see below), the colouring is perfect, thus $f \in H$, and $c(0) \neq c(1)$, thus $f$ interchanges the two colours. This makes $Q$ a homomorphism.

In general, there is no such homomorphism $Q$ : All elements $\pi \in H / K$ are of finite order. If $Q(\pi)$ contains a translational part, it is of infinite order in $H$. Thus there is $k=\operatorname{ord}(\pi)$ such that $\mathrm{id}=Q(\mathrm{id})=Q\left(\pi^{k}\right) \neq Q(\pi)^{k}$, hence $Q$ is not a homomorphism.

The elements $z \in \mathcal{M}_{n}$ with $|z|=1$ are exactly the $N$ elements of the form $\pm \xi_{n}^{i}$ (see Lemma 4.2). Thus they can carry at most $N$ colours. They can be mapped to each other by rotations about 0 , or by reflection $z \mapsto \bar{z}$. $H$ acts transitively on the colours. Thus in any colouring with more than $N$ colours there has to be a map $g \in H$ which is neither a rotation about 0 , nor a reflection $z \mapsto \bar{z}$. Thus the colouring requires a map $h$ with some translational part, which is of infinite order. Consequently, $Q(h)$ is of infinite order.

The results in this section yield $H$ in general - that is, whether a colouring of $\mathcal{M}_{n}$ is perfect or not - depending on the factorisation of the generator of the underlying ideal. In the next section we obtain results yielding $K$, depending only on the number $\ell$ of colours. As a byproduct, we also obtain partial results on $H$, depending on $\ell$ only.

\section{The Structure of $K$}

This section contains several lemmas which determine the structure of $K$ in all but finitely many cases. Recall that $\ell$ denotes the number of colours and $c(x)$ denotes the colour of $x$. We denote the group of translations by elements in $(q)$ by $T_{(q)}$. Note that $T_{(q)}$ is always contained in $K$.

Lemma 5.1. $\ell \geq 2$ if and only if $c(0) \neq c\left( \pm \xi^{i}\right)$ for all $i \leq n$.

Proof. $\ell=1 \Leftrightarrow(q)=(1) \Leftrightarrow(q)=\left( \pm \xi^{i}\right)$ for some $i \Leftrightarrow 0, \xi^{i} \in(q) \Leftrightarrow c(0)=c\left(\xi^{i}\right)$.

Let $\phi$ denote Euler's totient function.

Lemma 5.2. Each 2-colouring of $\mathcal{M}_{n}$ induced by $(q)$ is perfect. Moreover, $\ell=2$, if and only if $c\left( \pm \xi^{i}\right)=c\left(\xi^{j}\right)$ for all $i, j \leq n$, if and only if $K=T_{(q)} \rtimes D_{N}$.

Proof. Since $\ell=2$, we consider two cosets of $(q)$, namely, $(q)$ and $(q)+1$. Note that $2 \in(q)$ and $\pm \xi^{i} \in(q)+1$ for any $i \in \mathbb{Z}$. Consequently $\pm \xi^{i} \pm \xi^{j} \in(q)$, while $\pm \xi^{i} \pm \xi^{j} \pm \xi^{k} \in(q)+1$ for any $i, j, k \in \mathbb{Z}$, and in general: If $z=\sum_{i=0}^{\phi(n)-1} \alpha_{i} \xi^{i} \in \mathcal{M}_{n}$, with $\alpha_{i} \in \mathbb{Z}$, then $z \in(q)$ if and only if $\sum_{i=0}^{\phi(n)-1} \alpha_{i} \equiv 0 \bmod 2$, otherwise $z \in(q)+1$. Now, $\bar{z}=\sum_{i=1}^{\phi(n)-1} \alpha_{i} \xi^{n-i}$, and by conjugation of $z$, the sum $\sum_{i=0}^{\phi(n)-1} \alpha_{i}$ does not change. This implies that $z \in(q)$ if and only if $\bar{z} \in(q)$. Similarly, $z \in(q)+1$ if and only if $\bar{z} \in(q)+1$. Thus the reflection $z \mapsto \bar{z}$ maps $(q)$ to itself and $(q)+1$ to $(q)+1$. Hence the reflection is in $H$, and so the colouring is perfect. Furthermore it fixes the coloured pattern, and so is also in $K$.

From Lemma 5.1 follows that $c\left( \pm \xi^{i}\right) \neq c(0) \neq c\left(\xi^{j}\right)$ for all $i, j$. Since $\ell=2$, that is, there are two colours only, it follows $c\left( \pm \xi^{i}\right)=c\left(\xi^{j}\right)$.

Vice versa, if $c\left( \pm \xi^{i}\right)=c\left(\xi^{j}\right)$ for all $i, j$, then $\pm \xi^{i}-\xi^{j}, 2 \in(q)$. This means, analogous to the reasoning above, $(q)=\left\{\sum_{i=0}^{\phi(n)-1} \alpha_{i} \xi^{i} \mid \sum_{i=0}^{\phi(n)-1} \alpha_{i} \equiv 0 \bmod 2, \alpha_{i} \in \mathbb{Z}\right\}$, and thus $(q)$ has only one other coset, say $(q)+1$. This settles the first equivalence. Since $\pm \xi^{i}(q)=(q), \pm \xi^{i}((q)+1)=$ $(q) \pm \xi^{i}=(q)+1$, it follows that both the cosets are invariant under $N$-fold rotations, and so 
$K=T_{(q)} \rtimes D_{N}$, since the reflection is also in $K$ as noted above. Vice versa, if $K=T_{(q)} \rtimes D_{N}$, then $c\left( \pm \xi^{i}\right)=c\left(\xi^{j}\right)$.

Lemma 5.3. For all $\ell$-colourings of $\mathcal{M}_{n}$ holds: If $\ell>2^{\phi(n)}$ then $K=T_{(q)}$.

Proof. Recall that $K$ is a subgroup of $T_{(q)} \rtimes D_{N}$. If some id $\neq g \in D_{N}$ is an element of $K$, then $g$ maps some $\pm \xi^{i}$ to some $\xi^{j} \neq \pm \xi^{i}$, with $c\left(\xi^{i}\right)=c\left( \pm \xi^{j}\right)$. Thus it suffices to show $c\left(\xi^{i}\right) \neq c\left( \pm \xi^{j}\right)$ for all $i \neq j$.

Assume $c\left(\xi^{i}\right)=c\left( \pm \xi^{j}\right)$. Then $\xi^{i} \pm \xi^{j} \in(q)$. Hence

$$
N_{n}\left(\xi^{i} \pm \xi^{j}\right)=\left|\prod_{k=1}^{\phi(n)} \sigma_{k}\left(\xi^{i} \pm \xi^{j}\right)\right|=\prod_{k=1}^{\phi(n)}\left|\xi^{i_{k}} \pm \xi^{j_{k}}\right| \leq 2^{\phi(n)}, \text { where } \sigma_{k} \in \operatorname{Gal}\left(\mathbb{Q}\left(\xi_{n}\right), \mathbb{Q}\right) \text {. }
$$

It follows $\ell=\left[\mathcal{M}_{n}:(q)\right] \leq N_{n}\left(\xi^{i} \pm \xi^{j}\right) \leq 2^{\phi(n)}$, which contradicts $\ell>2^{\phi(n)}$.

Lemma 5.4. If $(q)=(2)$, then $H=G$. Furthermore, for all $n \neq 4$ in (11): $K=T_{(q)} \rtimes C_{2}$; and for $n=4: K=T_{(q)} \rtimes D_{2}$.

Proof. $q=2$ is balanced. Thus, $H=G$.

Now for $K$, compare the last proof: Note that $N_{n}(2)=2^{\phi(n)}$, and $c(1)=c(-1)$. But for all other complex roots of unity $\pm \xi_{n}^{k}(0<k<n)$ holds $c(1) \neq c\left(\xi_{n}^{k}\right)$, since otherwise we would have a factor of modulus strictly less than 2 in the equation above, and the $\leq$ becomes $<$. Consequently, $c\left(\xi^{i}\right)=c\left(-\xi^{i}\right)$, and so the only rotation in $K$ is the rotation by $\pi$ about 0 .

The reflection $z \mapsto \bar{z}$ maps $\xi$ to $\xi^{n-1}$. For all $n \neq 4$ in (1), $N_{n}\left(\xi-\xi^{n-1}\right)<2^{\phi(n)}$ and so $c(\xi) \neq c\left(\xi^{n-1}\right)$. Thus the reflection is not contained in $K$, and so $K=T_{(q)} \rtimes C_{2}$. Only for the case $n=4$ we get $N_{n}\left(\xi-\xi^{n-1}\right)=2^{\phi(n)}$, and so the reflection is in $K$ and thus $K=T_{(q)} \rtimes D_{2}$.

Why is the case $n=4, \ell=2^{\phi(4)}=4$ different? By inspection of this case (see Figure 1) we find that $K=T_{(2)} \rtimes D_{2}$. This is because $c(1)=c(-1)$ and $c(i)=c(-i)$; and only in this case does the reflection $z \mapsto \bar{z}$ also belong in $K$.

Lemma 5.5. If $\ell=2^{\phi(n)}$ but $(q) \neq(2)$, then $K=T_{(q)}$

Proof. From the proof of the previous lemma, there can be no more symmetries than the rotation by $\pi$ that can fix the colours. If this rotation by $\pi$ indeed fixes the colours, then in particular $c(1)=c(-1)$. Thus $2 \in(q)$, and so $(2) \subseteq(q)$. But since $(q)$ and $(2)$ have equal algebraic norms, then it follows that $(q)=(2)$, which is a contradiction. Therefore, $K=T_{(q)}$.

The case $\ell=2^{\phi(n)}$ but $(q) \neq(2)$ first occurs when $n=7$, see Table 1 .

Lemma 5.6. If $2<\ell=n$, where $n$ is prime in $\mathbb{Z}$, then $H=G$ and $K=T_{(q)} \rtimes D_{n}$.

Proof. Note that in the case when $n$ is an odd prime, the symmetry group of $(q)$ contains $D_{N}=$ $D_{2 n}$.

Let $2<\ell=n$ and $n$ prime in $\mathbb{Z}$. Then the unique factorisation of $\ell=n$ in $\mathcal{M}_{n}$ is $\ell=\prod_{i=1}^{n-1}\left(1-\xi^{i}\right)$ [17. Thus $\ell$ ramifies, and the possible generators of the ideal $(q)$ are exactly the $1-\xi^{i}$. Therefore, by Theorem 4.7, each corresponding colouring is perfect. In fact, there is only one such colouring, since for all $1 \leq j \leq n$ holds: $1-\xi^{j} \in(1-\xi)$. (This follows from $\xi^{k}(1-\xi) \in(q)$, thus $\sum_{k=0}^{j-1} \xi^{k}(1-\xi)=1-\xi^{j} \in(q)$.) Moreover, it follows that $c(1)=c\left(\xi^{j}\right)$ for all $j$.

Since $\ell$ is prime in $\mathbb{Z}$, we have $\mathcal{M}_{n} /(q) \cong C_{\ell}$, and so the $\ell$ distinct cosets can be expressed as $(q),(q)+1,(q)+2, \ldots,(q)+\ell-1$. Each coset is invariant under multiplication by $\xi^{j}$, but not under multiplication by $-\xi$. Thus, $K=T_{(q)} \rtimes D_{n}$.

Lemma 5.7. If $H=G$ and $\ell$ is prime in $\mathbb{Z}$, then $K$ contains a reflection. Thus, $T_{(q)} \rtimes C_{2}$ is a subgroup of $K$. 
E.P. BUGARIN, M.L.A.N. DE LAS PEÑAS, AND D. FRETTLÖH

Proof. Because $\ell$ is prime, the $\ell$ distinct cosets are $(q),(q)+1, \ldots,(q)+\ell-1$. Clearly, these cosets are invariant under conjugation, hence the reflection $z \mapsto \bar{z}$ is contained in $K$. Consequently, $K$ contains $T_{(q)} \rtimes C_{2}$ as a subgroup.

The previous lemma together with Lemma 5.3 yields the following result immediately.

Lemma 5.8. If $\ell$ is prime in $\mathbb{Z}$ and $K=T_{(q)}$, then $H=G^{\prime}$. In particular, if $\ell>2^{\phi(n)}$ is prime in $\mathbb{Z}$, then $H=G^{\prime}$.

Lemma 5.9. If $\ell>n$ and $\ell$ is prime in $\mathbb{Z}$, then $H=G^{\prime}$.

Proof. If $H=G$, then by Lemma 5.7 the cosets must be fixed by taking conjugates. This would mean that $c\left(\xi^{i}\right)=c\left(\xi^{n-i}\right)$ and so $\xi^{i}\left(1-\xi^{n-2 i}\right) \in(q)$. Thus $\ell=N_{n}(q) \mid N_{n}\left(1-\xi^{n-2 i}\right)=: \alpha$. Now, $\alpha$ must be either $2^{\phi(n)}$ (when $\xi^{n-2 i}=-1$ ) or a factor of $n^{\phi(n)}$. For the latter case, recall that $\prod_{j=1}^{n-1}\left(1-\xi^{j}\right)=n$. Taking the algebraic norm of both sides, and noting that this norm is completely multiplicative, gives us

$$
\prod_{j=1}^{n-1} N_{n}\left(1-\xi^{j}\right)=n^{\phi(n)}
$$

This suggests that each factor $N_{n}\left(1-\xi^{j}\right)$ on the left hand side of Equation (8) divides $n^{\phi(n)}$. But $\ell$ is not a prime factor of $n$, and so $\ell$ cannot divide $\alpha$. Hence $H=G^{\prime}$.

Lemma 5.10. If $\ell \nmid 2^{\phi(n)}$ and $\ell \nmid n^{\phi(n)}$, then $K=T_{(q)}$.

Proof. Suppose there is id $\neq g \in D_{N}$ which fixes the cosets, so in particular $g((q)+1)=(q)+1$. This implies that $(q) \pm \xi^{i}=(q)+1$ for some integer $i$, and hence $1 \pm \xi^{i} \in(q)$. As in the proof of the previous lemma, it follows then that $\ell \mid N_{n}\left(1 \pm \xi^{i}\right)=\beta$, where $\beta$ is either $2^{\phi(n)}$ or a factor of $n^{\phi(n)}$. This is a contradiction, thus $K=T_{(q)}$.

Table 1 illustrates applications of the results of the last two sections for the cases $n=3,4,7,9$. (These are exactly the values of $n$ where $\phi(n) \in\{2,6\}$. The cases $n=6,14,18$ are covered implicitly.) This table can be seen as a complement to Table 4 in [4, where values of $n$ for which $\phi(n)=4$ are considered. The entries in the second and third column follow from [2]. Many entries in the fourth and fifth column follow immediately from the results in this paper. Entries in brackets require further computations (compare [4), entries without brackets are immediate. Entries with an asterisk mean that there are multiple different possibilities. The last column lists one (out of possibly more than one) generator $q$ of a corresponding colouring. Note that for $n=7$, there are three colourings with 64 colours. The colouring induced by (2) is perfect, while the other two colourings are not.

\section{Application to quasiperiodic StruCtures}

Consider a colouring $c$ of $\mathcal{M}_{8}$ with eight colours. There exists exactly one such colouring [2]. By Corollary 4.5, this colouring is perfect. Therefore $H=\mathcal{M}_{8} \rtimes D_{8}$. Because of $N_{8}\left(1+\xi+\xi^{2}+\xi^{3}\right)=8$, this colouring is defined by the ideal $(q)=\left(1+\xi+\xi^{2}+\xi^{3}\right)$. Because of Theorem 4.4 and Lemma 4.3. $\bar{q}=1+\xi^{7}+\xi^{6}+\xi^{5} \in(q)$. Thus, $q+\bar{q}=2 \in(q)$. Hence the rotation by $\pi$ about 0 , which maps 1 to -1 , is contained in $K$. The rotation by $\pi / 2$ about 0 maps 1 to $i$. The norm of $1-i$ in $\mathcal{M}_{8}$ is $N_{8}(1-i)=4<8$, thus $1-i \notin(q)$. Therefore 1 and $i$ have different colours, and the rotation by $\pi / 2$ and thus the rotation by $\pi / 4$ are not contained in $K$. Finally, the reflection maps $\xi_{8}$ to $-\xi_{8}^{3}$. But $N_{8}\left(\xi_{8}+\xi_{8}^{3}\right)=4$ implying that $\xi_{8}$ and $-\xi_{8}^{3}$ have different colours. This yields $K=T_{(q)} \rtimes C_{2}$ (where $C_{2}$ represents the rotation by $\pi$ ).

Let us now describe how to illustrate this colouring $c$ and its symmetries. Since $\mathcal{M}_{8}$ is dense in the plane, we want a discrete subset of $\mathcal{M}_{8}$, which exhibits the colour symmetries of $\left(\mathcal{M}_{8}, c\right)$. A colouring of such a subset is shown in Figure 2. This set is well known from the theory of aperiodic 
TABle 1 . The cases $n=3,4,7,9$. Here, $j$ denotes the number of colourings with $\ell$ colours. Non-bracketed entries in the columns labelled $H$ and $K$ follow directly from results in this paper. Entries in brackets are computed by methods from [4].

\begin{tabular}{|c|r|c|c|c|c|}
\hline$n$ & $\ell$ & $j$ & $H$ & $K$ & $q$ \\
\hline 3 & 3 & 1 & $G$ & $T_{(q)} \rtimes D_{3}$ & $1-\xi_{3}$ \\
& 4 & 1 & $G$ & $T_{(q)} \rtimes C_{2}$ & 2 \\
\hline 4 & 2 & $*$ & $*$ & $T_{(q)}$ & $*$ \\
& 2 & 1 & $G$ & $T_{(q)} \rtimes D_{4}$ & $1-\xi_{4}$ \\
& $>4$ & 1 & $G$ & $T_{(q)} \rtimes D_{2}$ & 2 \\
\hline 7 & 7 & $*$ & $*$ & $T_{(q)}$ & $*$ \\
& 8 & 2 & $\left\{G^{\prime}\right\}$ & $\left\{T_{(q)} \rtimes C_{2}\right\}$ & $1-\xi_{7}-\xi_{7}^{3}$ \\
& 29 & 6 & $G^{\prime}$ & $T_{(q)}$ & $1-\xi_{7}-\xi_{7}^{2}$ \\
& 43 & 6 & $G^{\prime}$ & $T_{(q)}$ & $1-\xi_{7}-\xi_{7}^{2}-\xi_{7}^{3}$ \\
& 49 & 1 & $G$ & $\left\{T_{(q)}\right\}$ & $\left(1-\xi_{7}\right)^{2}$ \\
& 56 & 2 & $\left\{G^{\prime}\right\}$ & $T_{(q)}$ & $\left(1-\xi_{7}\right)\left(1-\xi_{7}-\xi_{7}^{3}\right)$ \\
& 64 & 1 & $G$ & $T_{(q)} \rtimes C_{2}$, & 2 \\
& & 2 & $\left\{G^{\prime}\right\}$ & $T_{(q)}$ & $\left(1-\xi_{7}-\xi_{7}^{3}\right)^{2}$ \\
& $>64$ & $*$ & $*$ & $T_{(q)}$ & $*$ \\
\hline 9 & 3 & 1 & $G$ & $\left\{T_{(q)} \rtimes D_{9}\right\}$ & $1-\xi_{9}$ \\
& 9 & 1 & $G$ & $\left\{T_{(q)}\right\}$ & $\left(1-\xi_{9}\right)^{2}$ \\
& 19 & 6 & $G^{\prime}$ & $T_{(q)}$ & $1-\xi_{9}-\xi_{9}^{2}$ \\
& 27 & 1 & $G$ & $\left\{T_{(q)}\right\}$ & $1-\xi_{9}^{3}$ \\
& 37 & 6 & $G^{\prime}$ & $T_{(q)}$ & $1-\xi_{9}-\xi_{9}^{3}$ \\
& 57 & 6 & $\left\{G^{\prime}\right\}$ & $T_{(q)}$ & $\left(1-\xi_{9}\right)\left(1-\xi_{9}-\xi_{9}^{2}\right)$ \\
& 64 & 1 & $G$ & $T_{(q)} \rtimes C_{2}$ & 2 \\
\hline 6 & $*$ & $*$ & $T_{(q)}$ & $*$ \\
\hline
\end{tabular}

order: It is the vertex set of an Ammann Beenker tiling, see [8] or [15]. The symmetries discussed above are visible in the image.

Further examples of perfect and chirally perfect colourings of quasiperiodic structures can be found in [9] (a 5-colouring of the vertex set of the famous Penrose tiling, based on $\mathcal{M}_{5}$ ), in [3] (an 8-colouring of a quasiperiodic pattern based on $\mathcal{M}_{7}$ ), in [10] (several colourings based on $\mathcal{M}_{n}$ for $n=4,6,8,10,12$ ), and in [4] (a 4-colouring of the Ammann Beenker tiling, based on $\mathcal{M}_{8}$ ). All these colourings arise from perfect or chirally perfect colourings of $\mathcal{M}_{n}$.

\section{Conclusion}

Two classical special cases of colour symmetries are covered by our approach, namely, the square lattice $\left(\mathcal{M}_{4}\right)$ and the hexagonal lattice $\left(\mathcal{M}_{3}\right.$, resp. $\left.\mathcal{M}_{6}\right)$. These are discrete point sets. In particular, we obtain Theorem 8.7.1 in [8] as a corollary, see Corollary 4.6. All other cases $(n=5$, $n \geq 7$ ) yield point sets $\mathcal{M}_{n}$ which are dense in the plane. In the case where $\mathcal{M}_{n}$ has class number one, we obtained our main results. These are a necessary and sufficient condition for a colouring to be perfect (Theorem 4.4). It allows the determination of the colour symmetry group $H$ of $\mathcal{M}_{n}$ in general. In particular, it yields all perfect colourings of $\mathcal{M}_{n}$. Moreover, for all but finitely many cases, we determine the subgroup $K$ of $H$ of symmetries which fix the coloured pattern: the lemmas in Section 5 aid the derivation of $K$. A systematic way to determine $K$ for a given ideal $\ell$-colouring would be to check the conditions of Lemma $5.2(\ell=2)$, Lemma $5.3\left(\ell>2^{\phi(n)}\right)$, Lemma 5.4 and Lemma $5.5\left(\ell=2^{\phi(n)}\right)$, Lemma 5.6 $\left(\ell=n\right.$ prime), Lemma $5.10\left(\ell \nmid 2^{\phi(n)}\right.$ and $\left.\ell \nmid n^{\phi(n)}\right)$. The remaining cases have to be handled individually. This allows — in principle — to obtain all colour preserving groups of (chirally) perfect colourings of $\mathcal{M}_{n}$. 


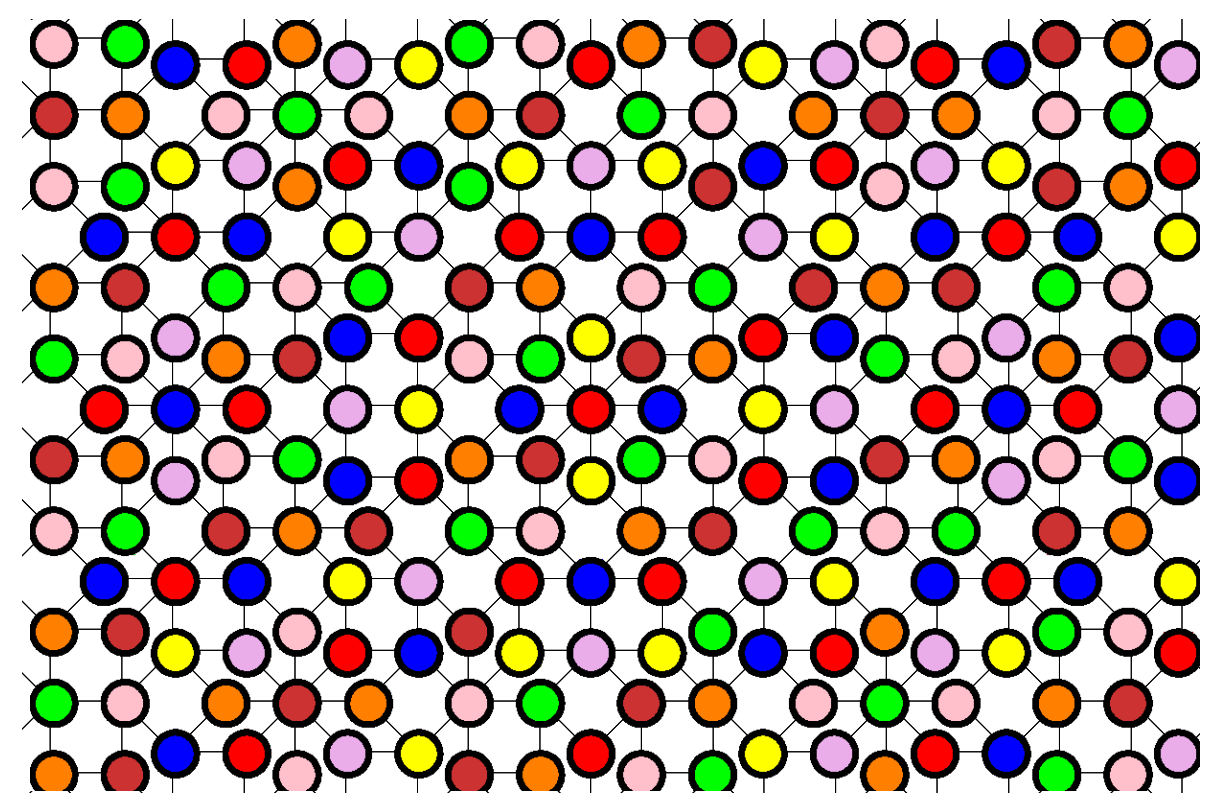

Figure 2. An 8-colouring of the vertices of the Ammann-Beenker tiling, arising from the 8-colouring of the underlying set $\mathcal{M}_{8}$.

For large $n$, the value $2^{\phi(n)}$ tends to be large, and it might be tedious to handle the remaining cases individually. Nevertheless, Lemma 5.10 seems to cover many of the remaining cases of $\ell$, compare Table 5 in 2. For instance, for $n=15$, there are 11 cases for which $\ell \leq 2^{\phi(15)}=256$. Our results cover 8 out of 11 cases, only three cases require further effort in order to derive the colour preserving group $K$. To give another example, for $n=16$, there are 23 cases for $\ell \leq 2^{\phi(16)}=256$, but 17 of them are covered by our results, and only six cases have to be checked individually in order to determine the group $K$.

\section{ACKNOWLEDGEMENTS}

The authors are grateful to Michael Baake and Christian Huck for helpful discussions. They wish to express their thanks to the CRC 701 of the German Research Council (DFG). The research leading to these results has received funding from the European Research Council under the European Union's Seventh Framework Programme (FP7/2007-2013) / ERC grant agreement no 247029 .

\section{REFERENCES}

[1] M. Baake: Combinatorial aspects of colour symmetries, J. Phys. A: Math. Gen. 30 (1997) 2687-98, mp_arc/02-323.

[2] M. Baake and U. Grimm: Bravais colourings of planar modules with N-fold symmetry, Z. Krist. 219 (2004) 72-80, math.C0/0301021.

[3] M. Baake, U. Grimm and M. Scheffer: Colourings of planar quasicrystals J. Alloys and Compounds 342 (2002) 195-197, cond-mat/0110654

[4] E.P. Bugarin, M.L.A.N. de las Peñas, I. Evidente, R.P. Felix and D. Frettlöh: On color groups of Bravais colorings of planar modules with quasicrystallographic symmetry, Z. Krist. 223 (2008), 785-790.

[5] J.H. Conway, H. Burgiel, C. Goodman-Strauss: "The symmetries of things", AK Peters, Wellesley (2008)

[6] M.L.A.N. de las Peñas, R.P. Felix and G.R. Laigo: Colorings of hyperbolic plane crystallographic patterns, $Z$. Krist. 221 (2006) 665-672.

[7] J. Dräger and N.D. Mermin: Superspace Groups without the Embedding: The Link between Superspace and Fourier-Space Crystallography, Phys. Rev. Lett. 76 (1996) 1489-1492.

[8] B. Grünbaum and G.C. Shephard: Tilings and patterns, Freeman, New York, 1987.

[9] R. Lifshitz: Theory of color symmetry for periodic and quasiperiodic crystals, Rev. Mod. Phys. 69 (1997) $1181-1218$ 
[10] R. Lück: Colour symmetry of 25 colours in quasiperiodic patterns, Phil. Mag. 88 (2008) 2049-2058.

[11] N.D. Mermin: Copernican crystallography, Phys. Rev. Lett. 68 (1992) 1172-1175.

[12] R.V. Moody and J. Patera: Colourings of quasicrystals, Can. J. Phys. 72 (1994) 442-452.

[13] R.L.E. Schwarzenberger: Colour symmetry, Bull. London Math. Soc. 16 (1984) 209-240.

[14] M. Senechal: Color groups, Discrete Appl. Math. 1 (1979) 51-73.

[15] M. Senechal: Quasicrystals and Geometry, Cambridge University Press (1995).

[16] B.L. van der Waerden and J.J. Burckhardt: Farbgruppen, Z. Krist. 115 (1961) 231-234.

[17] L.C. Washington: Introduction to cyclotomic fields, Springer, New York (1996).

Fakultät für Mathematik, Universität Bielefeld, 33501 Bielefeld, Germany

E-mail address: pbugarin@math.uni-bielefeld

Mathematics department, Ateneo de Manila University, loyola Heights, 1108 Quezon City, Philippines

E-mail address: mlp@math.admu.edu.ph

Institut für Mathematik, FU Berlin, 14195 Berlin, Germany

E-mail address: dirk.frettloeh@udo.edu 